\title{
Performance of the Resistive Plate Chambers as LVL1 ATLAS muon trigger detectors
}

\author{
M. Bianco, ${ }^{*}$ \\ on behalf of ATLAS Muon Collaboration \\ INFN Lecce, Physics Department, University of Salento \\ Lecce, 73100, Italy \\ *E-mail: michele.bianco@le.infn.it
}

\begin{abstract}
Resistive Plate Chambers (RPC) are used for the fast tracking trigger in the barrel region of the muon system which forms the outer shell of the ATLAS experiment at the Large Hadron Collider (LHC). This work reports on the results obtained with data taken during a combined run of Inner detector, Calorimeter and Muon system in 2009, where the trigger was provided by the $\mathrm{RPC}$. Results on RPC performance with respect to trigger alignment, coverage and efficiency using the entire ATLAS barrel trigger system will be presented. Finally, we review offline monitoring tools developed in the ATLAS software framework to provide fast feedback on RPC data quality.
\end{abstract}

\section{Introduction}

The ATLAS Muon Spectrometer uses Resistive Plate Chambers (RPC) detectors ${ }^{1}$ in the barrel region to trigger on muons and to identify the bunchcrossing. The system is made of $1116 \mathrm{RPC}$ units, which span different sizes and configurations covering a total surface of about $4000 \mathrm{~m}^{2}$.

The RPC detector and its first level trigger electronics are designed to detect and select high momentum muons and provide the bunch crossing identification, measuring the longitudinal ( $\eta$-view) and the azimuthal coordinates ( $\phi$-view) with a spatial resolution of $8-10 \mathrm{~mm}$. The RPCs are organized in several modules and their dimensions have been chosen to match those of the corresponding tracking precision chambers (MDT), to whom they are mechanically integrated. The so-called Middle Stations, at a radial distance of about $7 \mathrm{~m}$ from the interaction point contain two doublets of RPCs separated by $\sim 0.7 \mathrm{~m}$, called Confirm and Pivot doublets, while the so-called Outer Stations contain one doublet only, at $10 \mathrm{~m}$ radial distance. From the trigger point of view, the barrel system is segmented in 64 sectors, in the 
$\phi$ projection 32 sectors per half-barrel. Each physical chamber in the Pivot plane defines two trigger regions in the $\eta-\phi$ plane, called PADs, belonging logically to two trigger sectors, 396 PADs are installed in the whole ATLAS Barrel Muon Spectrometer. The trigger signal is generated inside the so called Coincidence Matrix (CMA) board, each PAD hosts 8 CMA.

\section{LVL1 trigger and timing performance}

The design of the Level-1 Muon Barrel has been driven by the simple concept of the pointing coincidence windows. ${ }^{2}$ The trigger is done with three planes segmented in strips in the $\mathrm{r}-\mathrm{z}$ and $\mathrm{r}-\phi$ projections. Using as origin the nominal position of the p-p interaction region, the strip hit in the Pivot plane of the Middle Station defines for low transverse momentum particles a coincidence window where to search for a correlated hit in the Confirm plane of the same Station (Low Pt algorithm). For higher momentum particles, then the strip hit in the middle plane defines also a new coincidence window where to search for a correlated hit in the Outer Station (High Pt algorithm). The trigger is done on both bending and non-bending projections. The three planes are made of two detector layers, and the order of the coincidence logic (2-out-of-4, 3-out-of-4, 4-out-of-4 majorities) is defined to maximize the efficiency and to minimize the rate due to uncorrelated and correlated background. During the commissioning phase, each tower was carefully tested and checked in order to assure the correct cabling, trigger response and the correct implementation of coincidence windows. In fig. 1 (b) the behaviour of the implemented trigger windows for a low $\mathrm{Pt} \phi$ Coincidence Matrix is shown.

A correct timing-in means that we will trigger the $\mu$, with the desired $\mathrm{Pt}$, emerging from the IP and we will stamp it with the correct Bunch Crossing (BC) ID. As decribed in section 1 the Muon Spectrometer in the barrel region consists of several elements, the timing-in of the trigger requires to correct for:

- The delay due to the propagation along cables, fibers and to the latencies of the different elements.

- The Time of Flight, i.e. the knowledge of the physical interesting configurations.

These delays have to be corrected in the pipelines of different elements. At the end of the timing process, the timing inside the same CMA, between CMAs within the same PAD, between PADs inside the same Trigger Sector and between different Trigger Sectors must be ensured. 
The best way to check it is to use only pointing tracks with known time of flight and look at relative timing. Dedicated runs were taken using Transition Radiation Tracker (TRT) ${ }^{3}$ as source of external trigger bucause its small radius allow to select pointing tracks easily. The misalignment between trigger towers inside same Sector Logic and between different Sector Logic have been significantly reduced via an iterative procedure.
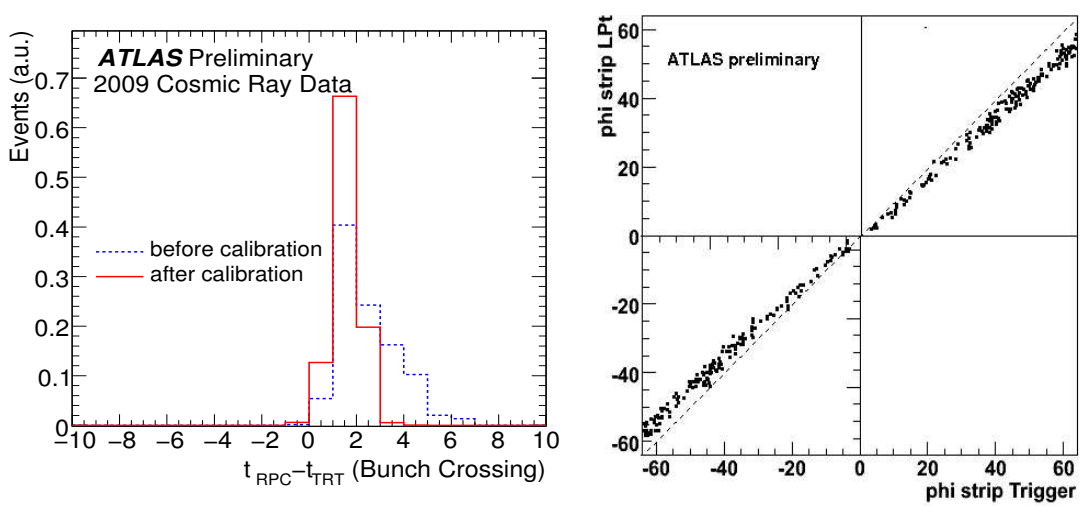

Fig. 1. RPC Trigger distribution wrt TRT trigger signal (a). Trigger windows for a low Pt $\phi$ Coincidence Matrix (b)

\section{RPC cosmic data analysis and results}

In order to ensure redundancy and robustness, a twofold strategy is used for RPC detector studies: Tracking with MDT detector and RPC StandAlone Tracking.

In the first case, (Tracking with MDT detector), muon tracks are built using the MDT detectors. Main advantage of this approach is that tracking and extrapolation to RPC layers correctly takes into account materials and magnetic field. The MDT intrinsec resolution allows to determine the RPC spatial resolution and to study small local effects. This is applicable only to runs when the tracking chambers were on. The tracks are selected requiring only one track per event, $\chi^{2} / d o f \leq 20$ and at least 2 hits in the $(\phi)$ view. In the second case,(RPC StandAlone Tracking), the pattern recognition is seeded by a straight line, which is defined by two RPC space points belonging, respectively, to Confirm and Pivot planes of the same or nearby station. RPC space points not part of any selected track and inside a predefined 
distance from the straight line are associated to the pattern. Resulting patterns with points in at least 3 out of 4 layers in Confirm and Pivot planes are retained and a linear interpolation is performed in two orthogonal views. From cosmic ray data about $95 \%$ percent of events have at least one RPC track; this is due to the strong correlation between the pattern recognition and the trigger algorithm.

Applying a quality cut on $\chi^{2} /$ dof about $70 \%$ of events have at least one good track and $10 \%$ more than one. The detection efficiency is measured by repeating 6 times the RPC tracking. At each iteration the layer under test is removed from the pattern recognition and track fitting. The track reconstructed is then extrapolated to the active gas volume of the removed layer and the crossing point evaluated. Studies to compare the two different methods are in progress for mutual validation. Until now there is no evidence for a difference between the methods.

\section{Monitoring and Data Quality status}

A software package to debug, monitor, and asses data quality for the RPC detector, has been developed within the ATLAS software framework (ATHENA). ${ }^{4}$ Being a part of the muon spectrometer offline monitoring package, the code runs automatically at the CERN computing facility (Tier0), where data are processed just after being available on the central data storage. Run by run, all relevant quantities characterizing the RPC detector are measured (such as efficiency, adjacent strip multiplicity, noise, ...) and stored in a dedicate database. These quantities are used for MonteCarlo simulations and offline reconstruction by physics analysis groups. The code was developed using $\mathrm{C}++$ objet oriented framework and it is configurable via Python script. Inside the Monitoring offline package, three algorithms have been developed, each focusing on specific monitoring task: RPC, RPCLV1, MDTvsRPC. The status of ATLAS data taking is monitored using the information from the data acquisition and trigger systems (TDAQ), and the analysis of events reconstructed online and offline is the basis for the Data Quality Assessment or DQA. DQA comprises data quality monitoring (DQM), it flags the data for future use in physics analysis. RPC system has three different sources of DQA: The Detector Control System (DCS), online and offline monitoring. Inside DCS, threshold on active fraction of the detector is applied to generate the Data Quality Assesment. Online and offline monitoring use the ATLAS DQM Framework to generate the DQA, it allows to apply automatically pre-defined algorithm to check reference histograms. DQA results are grouped as the DAQ partition are 
collected in specific DB. Offline Data Quality is actually totally based on $\mathrm{RPC}$ offline monitoring performed at Tier0.

\section{Conclusions}

RPC detectors have been installed and commissioned since long time and cosmic ray data taking allowed to perform a complete detector characterization. The RPC LVL1 trigger system is tested, and the timing alignment is almost complete. To perform a complete detector characterization two different offline strategies of detector performance analysis have been developed and tested, the characterization results are stored in dedicated DB and are ready to be used for the offline analysis. The RPC DataQuality system is in place and regularly monitors the quality of data during the runs.

\section{References}

1. R. Santonico and R. Cardarelli, Nucl. Instr. and Meth. A 187 (1981) 377.

2. ATLAS Trigger Performance Group, ATLAS Trigger Performance Status Report. CERN/LHCC/98-15, (1998).

3. ATLAS Collaboration. ATLAS Detector and Physics Performance Technical Desing Report. CERN/LHCC/99-15, 99-16, (1999).

4. ATLAS Computing Group, ATLAS Computing Technical Design Report. ATLAS TDR-017, CERN/LHCC/2005-022, (2005). 